\title{
SwissNET: le registre suisse de tumeurs neuroendocrines
}

\author{
Aurel Perren ${ }^{a}$, \\ Christoph Stettler $^{b}$ \\ a Prof. Dr, président SwissNET \\ b PD Dr, responsable \\ du registre
}

Traduction: Frédéric Triponez

\section{* Study nurse:}

Christiane Schwarzenbach, Universitätspoliklinik für Endokrinologie, Diabetologie und Klinische Ernährung, Inselspital, 3010 Berne, tél. 0316323038

Correspondance: SwissNET c/o Prof. Dr Aurel Perren Institut für Pathologie Universität Bern Postfach 62

CH-3010 Berne

Tél. 0316323222

Fax 0316324995
Les tumeurs neuroendocrines sont rares et les différentes spécialités médico-chirurgicales disposent de nombreuses possibilités de traitement. La rareté de ces tumeurs et la multiplicité des options thérapeutiques à disposition font qu'il n'y a guère d'évidence pour recommander une thérapie plutôt qu'une autre. Même les données épidémiologiques publiées sont lacunaires. La société SwissNET a été créée en décembre 2005. Elle réunit des spécialistes de différentes disciplines intéressés au traitement de tumeurs neuroendocrines, en particulier chirurgiens, endocrinologues, oncologues, médecins nucléaires, pathologues et gastroentérologues. Son but principal est la mise sur pieds d'un registre suisse de tumeurs neuroendocrines, tels qu'ils existent en France ou en Allemagne par exemple.

Afin d'avoir le recrutement le plus exhaustif que possible, SwissNET souhaite obtenir les données initiales de la part des instituts de pathologie, de façon analogue à ce qui se fait pour les registres cantonaux des tumeurs. Les données complémentaires et de suivi sont obtenues grâce aux médecins en charge du patient et en particulier du médecin traitant. Le succès du Registre est donc dépendant de votre collaboration.

\section{Les tumeurs neuroendocrines}

\section{sont rares et les différentes} spécialités médico-chirurgicales

\section{disposent de nombreuses}

\section{possibilités de traitement}

Les problèmes en lien avec la protection des données ont pu être résolus au niveau suisse puisque l'Office Fédéral de la Santé Publique a accordé à la Société SwissNET, dès février 2009, une autorisation spéciale pour la conduite du Registre. Un paragraphe de cette autorisation précise les buts du Registre: «Toutes les données des patients transmises au Registre suisse des tumeurs neuroendocrines ne peuvent servir qu'à l'enregistrement des sortes de tumeurs décrites dans l'autorisation. Il s'agit d'une saisie continue et systématique de données dans le but d'analyser et d'interpréter les données de toutes les personnes, si possible, atteintes de tumeurs neuroendocrines en Suisse. Le

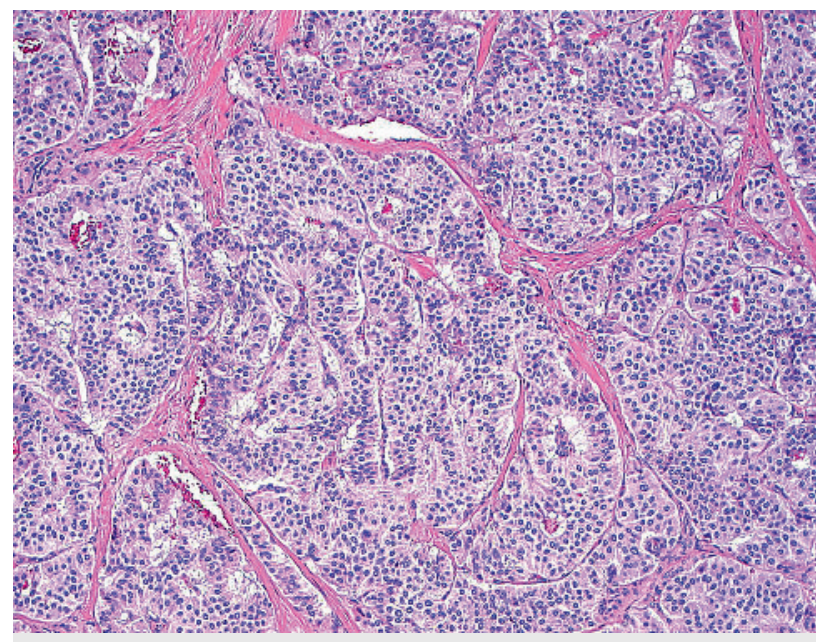

Cliché histopathologique d'un insulinome du pancréas.

Registre représente la base pour une recherche épidémiologique des tumeurs relativement rares que sont les tumeurs endocrines». Grace à cette «autorisation générale de lever le secret professionnel à des fins de recherche», tous les médecins, toutes les cliniques et instituts suisses sont autorisés à communiquer directement à SwissNET les noms et adresses des patients atteints de tumeurs neuroendocrines, afin qu'un consentement explicite puisse être obtenu du patient. Les patients sont informés de leur droit de refuser d'être intégré dans le Registre. Dans cette situation de refus du patient, aucune donnée clinique ne sera demandée aux différents intervenants médicaux.

Lorsque le patient accepte l'intégration de ses données (qui sont anonymisées) dans le Registre, toutes les données en rapport avec sa tumeur neuroendocrine et utilisées dans le but précédemment mentionné peuvent être transmises au Registre par tous les intervenants médicaux. De même, l'accès au dossier du patient est autorisé. Les données sans rapport avec la tumeur neuroendocrine ne peuvent pas être transmises.

Le Registre a besoin de votre soutien pour qu'il puisse mener ses tâches avec succès. La «study nurse»* engagée par le Registre s'occupe de contacter les instituts de pathologie ainsi que les médecins traitants. Nous restons à votre disposition si vous souhaitez avoir plus d'information. 